\title{
Innovation Qualité 2018 - les lauréats (2)
}

\section{Michelle Gerbera , Fabienne Hohlb}

a lic. phil. hum., collaboratrice scientifique de la FMH, division Données, démographie et qualité (DDQ) / Académie suisse pour la qualité en médecine (ASQM)

b Division Communication de la FMH

L'Innovation Qualité, le nouveau prix de l'Académie suisse pour la qualité en médecine (ASQM) de la FMH, a été remis pour la première fois le 17 avril 2018. Après avoir présenté les lauréats des catégories Repenser la prise en charge des patients et Sécurité des patients dans le numéro 18 du Bulletin des médecins suisses, nous avons aujourd'hui le plaisir de vous faire connaître les deux lauréats de la catégorie Organisations médicales.

\section{Décider intelligemment pour davantage de qualité de vie}

\author{
Prof. Dr méd. Jean-Michel Gaspoz ; Bernadette Häfliger Berger ${ }^{b}$ \\ a Président smarter medicine - Choosing Wisely Switzerland \\ ${ }^{b}$ Directrice smarter medicine - Choosing Wisely Switzerland
}

Il y a près de quatre ans, la Société suisse de médecine interne générale (SSMIG) a lancé l'initiative smarter medicine, largement inspirée par la campagne Choosing Wisely du corps médical américain. En 2014 et 2016, la SSMIG a été la première société de discipline médicale en Suisse à élaborer et publier des listes top 5 de mesures médicales ambulatoires ou hospitalières. Ces listes énoncent des traitements pour lesquels les soins sont soit superflus, soit excessifs en vue de sensibiliser les médecins mais aussi les patients à renoncer à ce qui apporte peu ou pas du tout de bénéfice à la santé mais limite la qualité de vie.

\section{Large alliance contre l'excès de soins}

Conjointement avec l'Académie Suisse des Sciences Médicales (ASSM), la SSMIG a développé l'idée d'une association smarter medicine en été 2016 afin de sceller
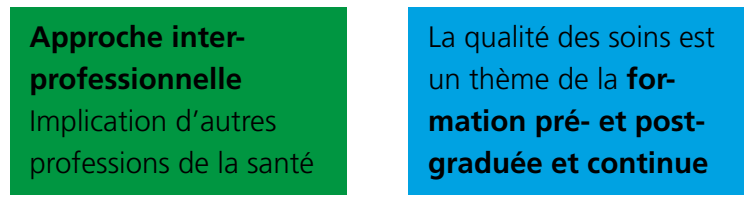

Sensibilisation des patients et des consommateurs

$$
\begin{aligned}
& \text { Soutien de la } \\
& \text { campagne par les } \\
& \text { politiques }
\end{aligned}
$$

Débat public sur les

soins superflus ou

excessifs (qualité du

traitement) 
Correspondance: Prof. Jean-Michel Gaspoz Médecine de premier recours

Rue Gabrielle-Perret-Gentil 4 CH-1205 Genève Tél. 0223729520 jean-michel.gaspoz[at] STOP-SPAM.hcuge.ch

Bernadette Häfliger Berger Société suisse de médecine interne générale

Monbijoustrasse 43

CH-3001 Berne

Tél. 0313704006

Bernadette.haefliger[at] sgaim.ch une large alliance entre le corps médical, les professions paramédicales, les organisations de patients et de consommateurs. L'association smarter medicine Choosing Wisely Switzerland a vu le jour début juin 2017.

\section{Communication entre patients et soignants}

Pour l'association, il est primordial que les recommandations soient élaborées directement par les sociétés de discipline et non édictées par les autorités politiques ou les assureurs-maladie. Les objectifs supplémentaires ont été définis conjointement avec tous les membres fondatuers (voir figure 1).

\section{Premiers succès et perspectives}

Dans la foulée de la fondation de l'association, plusieurs sociétés de discipline supplémentaires ont élaboré leurs propres listes top 5 et la publication d'autres listes est prévue prochainement.

Avec l'association, la SSMIG organise à Zurich du $1^{\text {er }}$ au 3 octobre 2018 un congrès international Choosing Wisely au cours duquel les experts du monde entier viendront présenter et échanger leurs nouvelles connaissances sur ce sujet.

Enfin, l'association prépare une campagne de sensibilisation pluriannuelle visant à familiariser le grand public à la question des soins superflus ou excessifs. Cette campagne prévoit entre autres des journées d'infor-

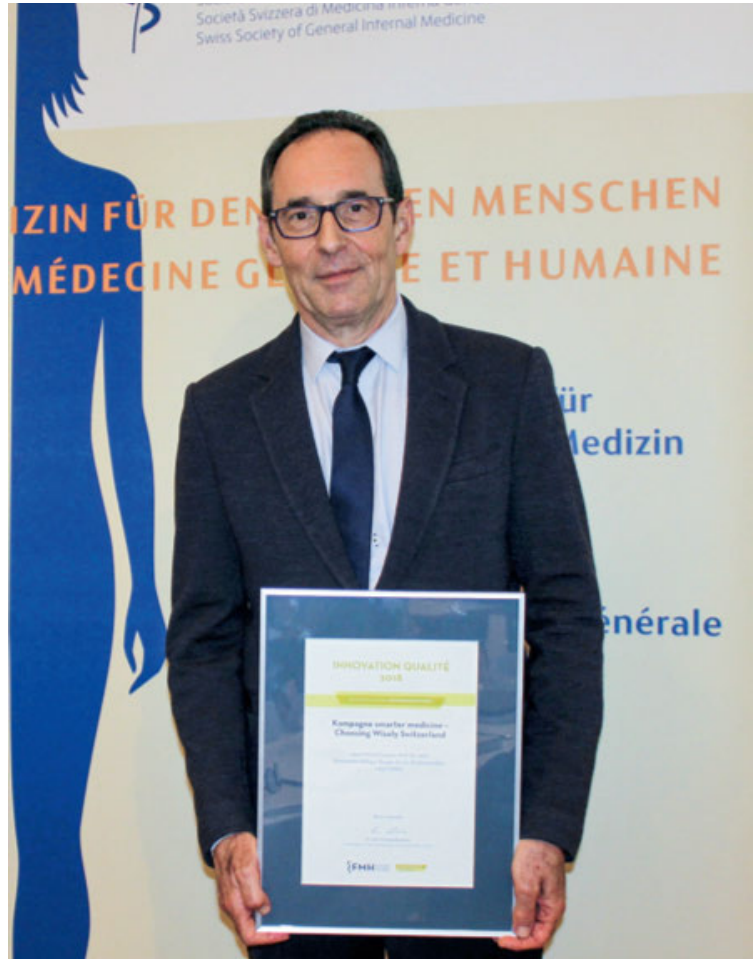

Le prix Innovation Qualité a été décerné a Jean-Michel Gaspoz.

mation et des outils d'information spécifiques pour les patients.

Pour en savoir plus sur l'initiative smarter medicine: www.smartermedicine.ch

\title{
Certification en oncologie - un projet de la SSOM
}

\author{
Walter Mingrone ${ }^{a}$, Christoph Renner $^{b}$, Ursula Kapp ${ }^{c}$, Volker Kirchner ${ }^{d}$, \\ Thomas von Briele, Kurt Beretta ${ }^{\mathrm{f}}$, Rudolf Morant ${ }^{\mathrm{g}}$, Markus Borner ${ }^{\mathrm{h}}$
}

${ }^{a}$ Dr méd., membre du comité de la SSOM et coprésident du Swiss Cancer Network (SCN); b Prof. Dr méd., coprésident du SCN;

${ }^{c}$ Prof. Dr méd., membre du comité du SCN; ${ }^{d}$ Dr méd., coprésident de la SSOM et membre du comité du SCN;

${ }^{e}$ Dr méd., membre du comité du SCN; ${ }^{\dagger}$ Dr méd., membre du comité de la SSOM et du SCN;

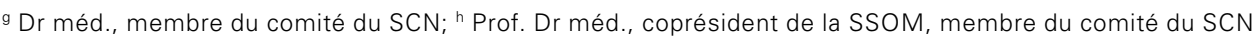

Contexte et objectifs. Il y a près de 10 ans, plusieurs cliniques de Suisse alémanique ont commencé à obtenir des certifications selon les critères de sociétés étrangères. En parallèle, les premières certifications par organes voyaient le jour, pour les centres du sein par exemple. Jusqu'ici, de très nombreux oncologues et patients n'avaient pas accès à un modèle de certification multiorgane tenant compte du fait que notre prise en charge est en grande partie décentralisée et assurée par des cabinets privés ou des centres ambulatoires hospitaliers.

Dans le cadre du programme national contre le cancer 2011-2015, la Société suisse d'oncologie médicale (SSOM) a créé son propre programme de certification afin de contribuer à ce que tous les patients puissent accéder à des soins oncologiques de qualité élevée proches de leur lieu de résidence.

Marche suivie. En décembre 2012, la SSOM a déve- 
loppé, conjointement avec la Société suisse de radio-oncologie et Soins en Oncologie Suisse, un concept de qualité commun accepté par la grande majorité de ses membres. La SSOM a ensuite fondé l'association Swiss Cancer Network (SCN), chargée notamment de développer un programme de certification sur la base du concept de qualité. Avant son implémentation, le programme a été lui aussi soumis à une votation générale en janvier 2014 auprès des membres de la SSOM, qui l'ont approuvé à $87 \%$.

Ce qu'atteste le certificat. Le certificat SCN met l'accent sur les recommandations de traitement fondées sur les preuves incluant une prise en charge interdisciplinaire et interprofessionnelle. Les mesures à observer suivent une approche axée sur les processus et respectent l'approche concurrentielle de notre pays.

La certification comprend cinq dimensions: qualification du personnel spécialisé; forme et contenu du tumor board y c. compte rendu; bases scientifiques de la prise en charge individuelle («lignes directrices»); collaboration au sein du réseau de soins et avec les institutions sociales; accès aux résultats thérapeutiques (pour en savoir plus: www.sgmo.ch $\rightarrow$ Assurance qualité $\rightarrow$ Swiss Cancer Network).

Certifications actuelles. Pour l'heure, plus de 30 institutions, des cabinets particuliers mais aussi des centres ambulatoires hospitaliers plus grands, situées aux quatre coins du pays sont certifiées conformément à leur autodéclaration. En association avec une société d'audit et avec l'intervention de deux experts spécialisés (des oncologues confirmés formés à la réalisation d'audits), cinq institutions ont déjà fait l'objet d'un audit sur place. La SSOM augmente en permanence le nombre d'audits.

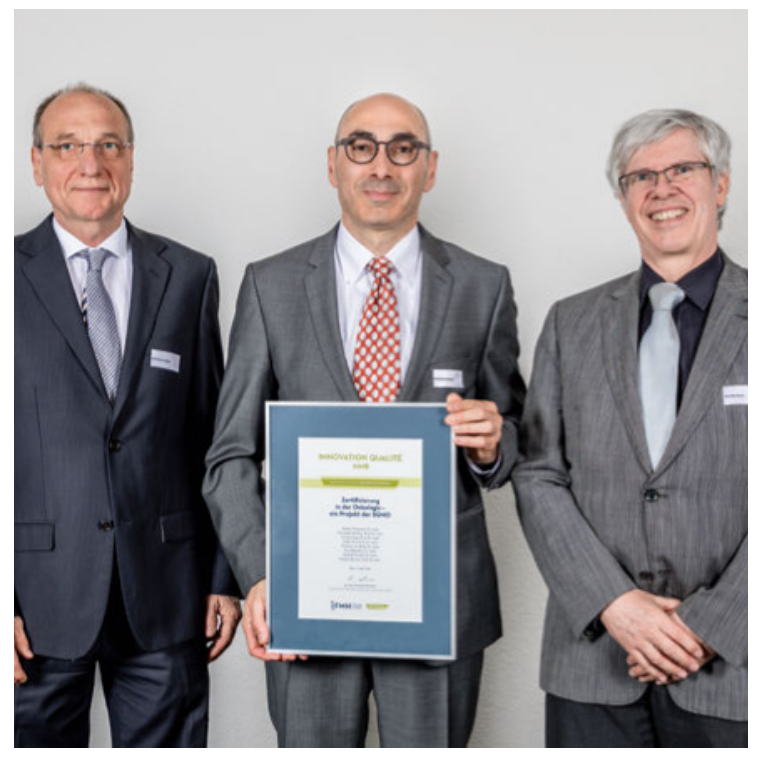

Volker Kirchner, Walter Mingrone et Kurt Beretta se réjouissent d'avoir obtenu le prix Innovation Qualité.

Ce dont nous sommes fiers. Conjointement avec les représentants de cabinets privés d'oncologie, de centres ambulatoires hospitaliers régionaux mais aussi avec des collègues d'hôpitaux universitaires, la SSOM a réussi à créer, de sa propre initiative - et à sa plus grande satisfaction -, un programme de certification permettant de garantir aux patients des soins oncologiques de qualité élevée. De plus, ce programme est adapté à notre contexte national et tient compte des habitudes de la prise en charge médicale en Suisse. La SSOM continuera à évaluer et à développer la certification en concertation avec ses membres. 